\title{
Assessment of the Ultimate Actual Strength of Rock-Climbing Protection Devices: Extraction Tests in the Field and the Human Capability to Predict the Ultimate Strength
}

\author{
A. Manes, F. Cadini \\ Politecnico di Milano University, Department of Mechanical Engineering, Milan, Italy
}

\author{
CORRESPONDING AUTHOR: \\ Andrea Manes \\ Politecnico di Milano University \\ Department of Mechanical Engineering \\ Via la Masa 1 \\ 20156, Milan, Italy \\ E-mail: andrea.manes@polimi.it
}

\section{DOI:}

10.32098/mltj.02.2020.09

LEVEL OF EVIDENCE: 4

\begin{abstract}
SUMMARY
Background. Rock climbing protection devices are crucial for climbing practice safety and for mountaineering in general. The use of these devices, together with appropriate techniques, reduces injuries in the critical event of a climber's fall. Although European standards and rules support the manufacturer in the design, production and laboratory testing, a thorough investigation of their behaviour in a real environment and during an actual placement has not yet been performed.

Methods. The aim of this work is to present an insight into the strength of such devices through the application of a monitored, quasi-static, increasing force in a field environment. Results from several types of devices (pitons, nuts and cams) are presented and critically evaluated with respect to the values of the loads acting on the anchors due to the fall of the climber.

Results. As far as the piton actual strength is concerned, the present activities show that the characteristics requested by EN specifications and rules are functional for product qualification purposes, but of very little use when defining the load holding capabilities once the devices are in place. However, even if the actual strength does not match the requirement of the standard, the comparison with the actual load applied is fairly encouraging. With regards to nuts and cams, it is worth underlining the importance of a correct placement: when placed correctly, the actual strength achieved by the device in the field complies and is higher than the classification of the EN standard. Moreover, an investigation of human capability to predict the ultimate strength of rock-climbing protection devices placed in the field has been carried out, with the aim of verifying the reliability of the climber's judgement, and, possibly, improve the safety of the in-field decision-making process.

Conclusions. The lesson learned from the experiments is that modern equipment shows one step better behaviour and, similarly to pitons, the device-rock coupling dictates the pairs actual strength, assuming of course a sound placement. To the author's best knowledge, the present work represents the first attempt to investigate the human capabilities to assess the reliability of a protection placement in-field.
\end{abstract}

\section{KEY WORDS}

Rock-climbing protection, actual strength, human prediction

\section{BACKGROUND}

While climbing a long rock or ice route, mountaineers are tied by means of a rope. The climb is subdivided into "pitches" by "stances" (or belay stances), i.e., places where anchoring points are available for security and for the mutual belay of the partners. Focusing on the security task, the anchors are used as 'runners' as the climber, one of the partners, 'lead climbs' higher from one stance to the next one. Note that from now onwards, the terms "anchor" and "protection" will be equivalently used to indicate the safety devices that are placed in the rock to protect the climbers (both for creating a stance and as a 'runners'). 
When the climber falls in a leading situation, the fall length is approximately twice the distance of the leader to the closest runner; the runner is therefore a constrain of the rope (and the mountaineers) to the rock or ice wall. This scenario can clearly produce severe loading on the anchors, as well as the possibility that the anchors fails, thus reducing the points of attachment of the rope on the rock wallpotentially critical for the safety of the mountaineers. In order to reduce the intrinsic risk of a complete detachment of the rope and mountaineers, the stance is built as a system of multiple anchor points, which are usually interconnected to increase safety. For several reasons, in case of a fall of a climber, the magnitude of the load on the stance is sensibly lower (as described in more detail below), and the multiple anchor system works synergistically in order to avoid catastrophic detachment of all the partners from the wall. A brief description of modern climbing methods can be found in (1). It is worth mentioning that protection devices are supposed to work together with other mountaineering equipment, specifically with an elastic rope that provides a limited reaction force to gradually stop the climber in case of a fall. The formerly UIAA 101 (2), followed by the formerly EN-892 (3) requires a peak force of the rope during testing lower than $12 \mathrm{kN}$. This is the peak force registered by the rope during a dynamic test in laboratory conditions. This condition is representative of an almost worst case. In the field, several factors reduce the maximum load to a level closer to $5-7 \mathrm{kN}$, as also discussed in the present article. As far as the stance is considered, a lower load can be considered on the single anchors of about 2-4 $\mathrm{kN}$ (4). The aforementioned equipment, including several others not described here, work like a safety chain. The safety chain is a concept developed by the UIAA (and followed by EU standards) to describe and harmonize all the pieces of equipment in climbing (rope, harness, carabiners, protections etc.). Standards provide requirements for all these components of the safety chain, so that they all work together in an integrated way in order to avoid catastrophic consequences in case of a fall.

Several types of rock-climbing protection devices are currently used, such as bolts (adhesive and friction expansion rock anchors), pitons, passive devices (tapers and camming chocks/nuts) and active devices (spring loaded camming devices also known as frictional anchor and called "friends" or "cams" in climber's jargon). European standards and rules provide design and strength requirements to the manufacturers, but the compliance verification tests are carried out in laboratories and do not consider several important issues: different types of rock (different strengths and different friction coefficients between the rock and the device), different shapes of cracks (where the devices should be placed), the users' ability of the placement, etc. Several climbing routes, especially modern ones, are equipped with chemically and/or friction bonded rock-climbing anchors called bolts. The strength of these devices is generally orders of magnitude larger than other types of protections (5) and several studies are available as similar anchors are used in civil engineering. These anchors are placed by means of drilling an artificial hole inside the rock, are permanent, and thus are less sensitive to variability due to placements. Some concerns arise for the permanent placement under aggressive environmental conditions (6). However, these conditions are not of interest for the present study. With regards to pitons, passive devices and active devices, their strength is strongly dependent upon the placement in the field. Due to the fact that their use is crucial for climbing practice and for mountaineering safety in general, an insight into the actual strength of such devices is critical in order to reduce injuries in the event of a fall of a climber.

Realistically simulating the force exerted on climbing equipment due to the fall of a lead climber is very difficult to achieve. Consequently, there are not any proven methods within the current literature for verifying the behaviour of rock climbing in such events. A methodology suitable to investigate these issues in a real environment, as well as to measure the strength both in terms of maximum load and failure analyses, could be of great interest in order to critically assess the equipment strength and also the correct procedures for the placement. Correct placement is fundamental in achieving the maximum strength of an anchor when withstanding the load generated during fall.

Although the actual load application is dynamic, the European standards refer to static tests to verify the compliance to the requested strengths. While static versus dynamic tests is a point to be discussed, it seems reasonable to neglect the strain rate effect, and the dynamic influence on the behaviour of the devices due to impact velocity is relatively low (although for spring loaded camming devices this subject needs more accurate evaluations for the dynamic behaviour induced by the spring).

In this work we exploit a thorough in-field experimental campaign conducted by a team of expert climbers, some of which also have a structural/mechanical engineering background. This allows to better understand the actual behavior of these devices and, possibly, to highlight how the type of device (material and geometry) and the boundary conditions in which they operate (cracks in rock material) affect their in-field protection capability (i.e., their ultimate strength). The strength of these protection devices was tested through the application of a monitored, quasi-static, increasing force in a field environment. 
Moreover, dynamic falling tests were also performed both in laboratory environments and in the field. These tests were only aimed at measuring the load at the level of the anchor, but not the anchor strength (in most of the cases the anchors used in these tests were dummies with a very high strength and therefore had no possibility of failing during the application of the load). These tests involved dropping a weight mimicking a fall of the climber from a given height and measuring the load on the last protection and/or on the stance of the anchor/safety chain.

The load data collected in both types of tests were statistically processed to infer (i) the behaviour of the different protection devices in different operative conditions, and (ii) to estimate a trend of the load that was applied to such devices, to be able to identify the most critical conditions. The proposed statistical framework estimates the probability of device failure based on the conditions of a fall. Results will show that the strength of the anchors depends on the limit value of the anchor, the rock type, and the placement, in combination with a large variety of loading conditions that act upon the anchor.

In addition, experts were polled on their predictions of the failure loads before the trial actually took place. These data were used to investigate how capable experts would be at predicting the strength of rock-climbing protection devices before failure based on the testing conditions. Comparing the objective test results and the subjective expert estimations, the decision-making process can be improved in a real climbing environment. A total of 106 extraction tests were performed and more than 1000 predictions made.

\section{EXPERIMENTAL TESTS: METHODS}

Static tests were carried out on pitons, chocks and cam devices placed in a real environment and loaded by means of an oleo-dynamic piston, (figure 1). This system can be easily attached to any rock (or ice) surface and is able to apply a parallel-to-wall load by means of a common actuator. A gauged uniaxial load cell (50 KN rated) was used and the peak value was recorded (blue box, figure 1). The load cell was placed between the cylinder and the anchor, recording the collapse load. The anchor is shown inside the red circle in figure 1. Care was taken to avoid tangential strain to the cell. The hydraulic piston was fixed with a chain and pulley system to allow free movement of the piston alignment on the loading action line. The load application rate was set at a few $\mathrm{mm} / \mathrm{s}$ and took roughly 10-20 seconds for the complete collapse. The cylinder was controlled by an oleo-dynamic system fed by a pump driven both electrically and manually. The tests were conducted on several types of rock walls, including both hard rock (porphyry and granite) and soft rock (sandstone and dolomite). Bedogni and Manes, used a similar device for the assessment of ice screws in the field (7). Hard and soft rock are both typically found in climbing activities, however, different failure modes are expected. The expected failure mode of anchors in hard rock is more often dependent upon the failure of the device, whereas the failure mode in presence of soft stone potentially involves both the failure of the anchor system and the rock itself. The loading mechanism imposed by the piston is "displacement dependent" (in actual falls it is "load dependent"), therefore a temporary load decrease is possible to some extent.

An advantage of using a quasi-static system is that is makes it possible to ensure a system failure with every test. When a dynamic test is applied, the force is driven by the fall event

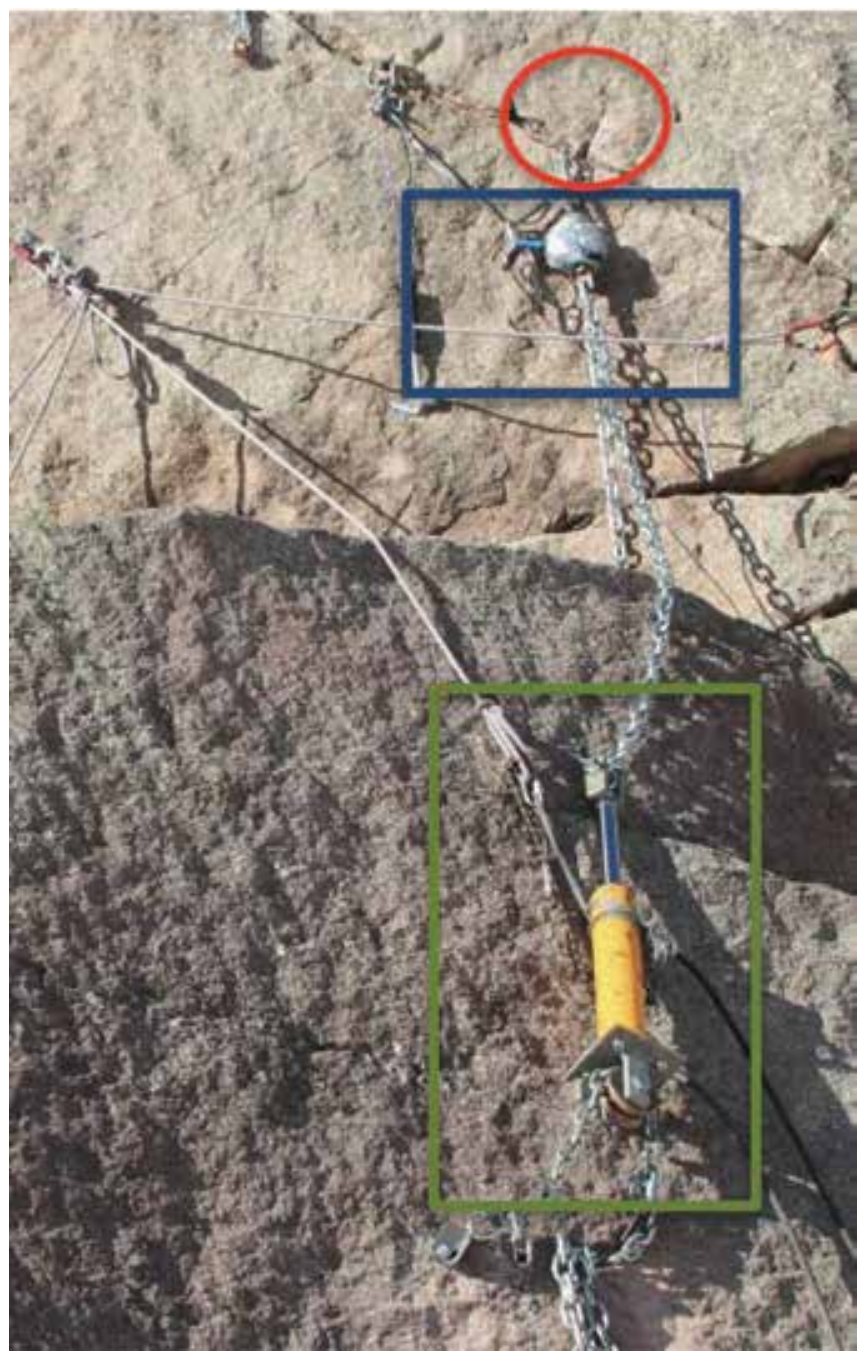

Figure 1. Extraction tests: the oleo-dynamic piston in the green box, a gauged uniaxial load cell (50 KN rated) in the blue box, the specimen placed inside the red circle. 
and it is possible that the safety restraint does not fail. Additionally, albeit the drop test reproduces more realistically an actual fall, dynamic tests present multiple logistical difficulties. Vogwell and Minguez (1) carried out drop tests in a laboratory environment placing anchor nuts in a standard simulated crevice device. Nevertheless, they had to use a standard tensile testing machine in order to determine the ultimate failure load of the anchor because the drop tests failed to break the system.

Tests consisted of a first phase devoted to the placement of the anchors in the field and a second phase of load application. It is essential that the anchor is properly fit to a rock crevice for optimal testing, as the results are dependent upon device placement. Devices were placed by mountaineering instructors and/or mountaineering military corps members: to ensure that the anchors were optimally installed.

In addition to the tests described above, each qualified person attending the tests was also required to make an informed prediction of the failure load of each device before testing.

The purpose was to obtain the predictions of the experts in order to investigate human capability to predict the ultimate strength of rock-climbing protection devices placed in the field. Experts were selected among qualified mountaineering instructors inside the Italian Alpine Club (CAI) and the Corps of the "Guardia di Finanza".

Table I shows the appearance of the piton failure load database. In particular, the first three columns report the specific features of each trial, i.e., the device material (only for pitons, column 1: pitons are usually built using soft steel, $\mathrm{S}$, and high carbon hardened steel, $\mathrm{H}$ ), the piton length (only for pitons, column 2) and the rock type (column 3): hard rock, $\mathrm{H}$ (porphyry and granite), and soft rock, $\mathrm{S}$ (sandstone and dolomite), while column 4 reports the observed failure load. At the same time, the columns from the sixth of the expert climber's predictions for each trial are shown when available.

\section{DATA ANALYSIS APPROACHES}

The first part of the data analysis was aimed at analyzing the behavior of the protection devices placed in field and comparing the failure loads observed for different protection devices (function, geometry and material) in different operative rock material. In order to perform this analysis, we opted to resort to a probabilistic framework, which naturally allows to account for all the uncertainties involved in the protection placement and the measurement processes, as described in the following.

The statistical analysis of the data collected assumes that the $N$ measurements available for each observed quantity are realizations of random variables accounting for all the uncertainties involved in the process. The analysis was initially based on the calculation of the empirical density function of these random variables, which approximates the (unknown) underlying probability density function:

$$
f^{\wedge}{ }_{N}(x)=\frac{1}{N} \sum_{i=1}^{N} \delta\left(x-x_{i}\right)
$$

where $N$ is the number of measurements, $x$ is the generic random variable (i.e., the failure loads or the dynamic load on the last protection), $x_{i}$ is its $i^{\text {th }}$ available realization (measurement) and $\delta$ is the Dirac function. Operatively, the empirical density $f^{\wedge}{ }_{\mathrm{N}}(x)$ can be computed by creating a properly normalized histogram of the available measurements. In this work, histograms of the different loads analyzed were

Table I. Piton failure load database.

\begin{tabular}{|c|c|c|c|c|c|c|c|c|c|c|}
\hline $\begin{array}{l}\text { Piton material } \\
\text { (Hard - Soft) }\end{array}$ & $\begin{array}{l}\text { length } \\
(60-80)\end{array}$ & $\begin{array}{c}\text { Rock type } \\
\text { (Hard - Soft) }\end{array}$ & $\begin{array}{c}\text { Failure load } \\
\text { (kgforce) }\end{array}$ & Reference & \begin{tabular}{|c|} 
Prediction \\
1 \\
\end{tabular} & \begin{tabular}{|c|} 
Prediction \\
$\mathbf{2}$ \\
\end{tabular} & \begin{tabular}{|c|} 
Prediction \\
3 \\
\end{tabular} & \begin{tabular}{c|} 
Prediction \\
4 \\
\end{tabular} & $\begin{array}{c}\text { Prediction } \\
\mathbf{5} \\
\end{array}$ & $\begin{array}{c}\text { Prediction } \\
6 \\
\end{array}$ \\
\hline $\mathrm{H}$ & 70 & $\mathrm{H}$ & 360 & $11 \mathrm{Val}$ masino 2012 & 700 & 500 & 800 & 400 & 450 & 400 \\
\hline $\mathrm{H}$ & 70 & $\mathrm{H}$ & 120 & $12 \mathrm{Val}$ masino 2012 & 1200 & 800 & 1300 & 900 & 1000 & 1500 \\
\hline $\mathrm{H}$ & 110 & $\mathrm{H}$ & 634 & $16 \mathrm{Val}$ masino 2012 & 1200 & 1000 & 1000 & 1200 & 1050 & 1000 \\
\hline $\mathrm{H}$ & 70 & $\mathrm{H}$ & 870 & $19 \mathrm{Val}$ masino 2012 & 900 & 600 & 800 & 900 & 1200 & 1000 \\
\hline $\mathrm{H}$ & 30 & $\mathrm{H}$ & 410 & $20 \mathrm{Val}$ masino 2012 & 300 & 200 & 300 & 300 & 150 & 300 \\
\hline $\mathrm{H}$ & 80 & $\mathrm{H}$ & 1857 & 2 Passo Rolle 1 & 900 & & 950 & 750 & 950 & 1200 \\
\hline 5 & 65 & $\mathrm{H}$ & 927 & $13 \mathrm{Val}$ masino 2012 & 900 & 700 & 800 & 800 & 950 & 1000 \\
\hline $\mathbf{s}$ & 50 & $\mathrm{H}$ & 1200 & $15 \mathrm{Val}$ masino 2012 & 500 & 400 & 700 & 800 & 650 & 350 \\
\hline $\mathbf{s}$ & 90 & $\mathrm{H}$ & 2227 & $17 \mathrm{Val}$ masino 2012 & 400 & 500 & 700 & 800 & 600 & 350 \\
\hline $\mathbf{s}$ & 60 & $\mathrm{H}$ & 100 & $21 \mathrm{Val}$ masino 2012 & 300 & 300 & 400 & 200 & 400 & 300 \\
\hline $\mathbf{s}$ & 90 & $\mathrm{H}$ & 567 & 1 Passo Rolle 1 & 950 & 600 & 750 & 1800 & 1200 & 800 \\
\hline $\mathbf{s}$ & 110 & $\mathrm{H}$ & 1210 & 3 Passo Rolle 1 & 300 & 1600 & 850 & 750 & 650 & 450 \\
\hline $\mathbf{s}$ & 80 & $\mathrm{H}$ & 1147 & 4 Passo Rolle 1 & 400 & 400 & 390 & 600 & 380 & 400 \\
\hline $\mathbf{s}$ & 110 & $\mathrm{H}$ & 1723 & 8 Passo Rolle 1 & 950 & 2000 & 1550 & 1400 & 1800 & 1300 \\
\hline $\mathrm{H}$ & 80 & 5 & 566 & 1 Passo Rolle 2014 & 280 & 400 & 300 & 300 & 250 & 300 \\
\hline $\mathrm{H}$ & 60 & 5 & 997 & 4 Passo Rolle 2014 & 900 & 800 & 1000 & 1100 & 1500 & 1100 \\
\hline
\end{tabular}


created by dividing a range of forces between 0 and 2500 $\mathrm{Kg}$ force into $20 \mathrm{bins}$; then, the number of measurements belonging to each bin was divided by the total number of observations $N$ and by the width of the bin $w_{b}$ (i.e., $w_{b}=$ $125 \mathrm{Kg}$ force). The number of bins was chosen on the basis of a trial and error procedure aimed at finding a trade-off between the statistical significance of the estimates and the number of measurements available. Note that the outcome of this procedure is a function $f^{\wedge}{ }_{\mathrm{N}}(x)$ known only at 20 given points, e.g., at the bin centers $x_{c}=\frac{w_{b}}{2} \cdot c, c=1, \cdots, 20$. Thus, in order to be able to compare the different empirical densities obtained more easily, the discrete functions $f^{\wedge}{ }_{\mathrm{N}}(x)$ were "smoothed" using kernel density estimator (8):

$$
f^{\wedge}{ }_{K}(x)=\frac{1}{N h} \sum_{i=1}^{N} K\left(\frac{x-x_{i}}{h}\right)
$$

where $\mathrm{K}$ is a non-negative function called kernel and $\mathrm{h}>0$ is a smoothing parameter, called bandwidth. Several choices for the kernel function are possible: here we restricted our attention to the popular case of $\mathrm{K}(\cdot)=\Phi(\cdot)$, where $\Phi(\cdot)$ is a Standard normal density function. Intuitively, the kernel density method requires that each realization $x_{i}$ (measurement) available of the random variable $X$ to be associated with a Standard Gaussian distribution with a mean equal to the realization $x_{i}$ itself and a variance equal to 1 . The band-

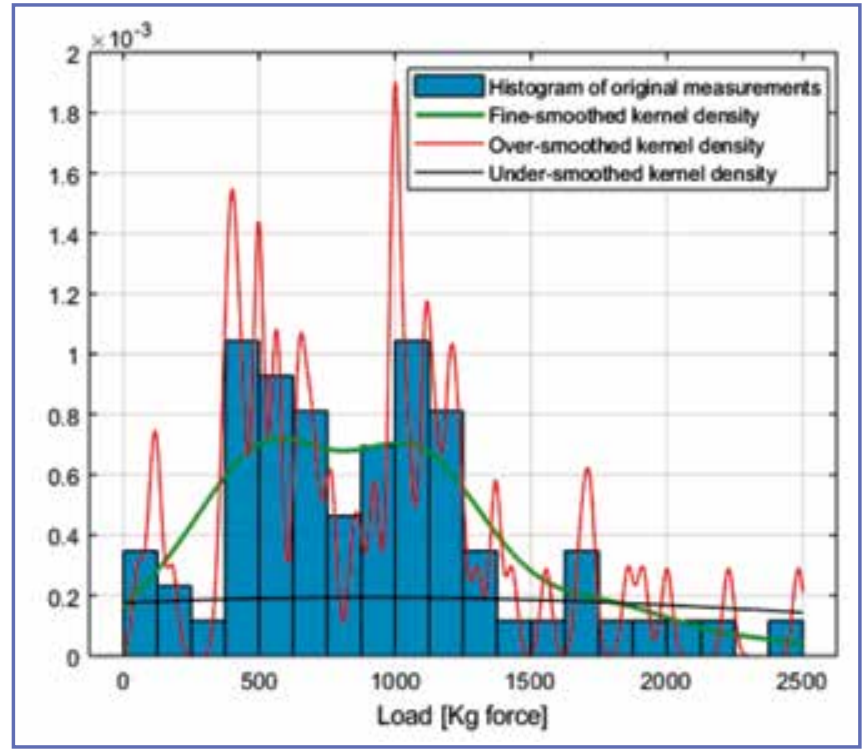

Figure 2. Histogram of the original static failure loads of all the pitons tested during the experimental campaign (light blue columns). Examples of kernel densities with different bandwidths $h=20$ (red line, over-smoothed), $h=200$ (green line, well-smoothed) and $h=2000$ (black line, under-smoothed). width $b$ allows tuning the smoothness of the resulting kernel density estimator $f^{\wedge}{ }_{\mathrm{N}}(x)$, as shown in the reference example of figure 2 .

The second part of the data analysis was focused on the evaluation of the quality of the experts' predictions of the static failure loads, in order to possibly identify common systematic errors and/or misinterpretations affecting the climber's judgement when practicing in the field. Due to the scarcity and sparsity of the available predictions (not all predictors were always present at each experimental session), we chose to compare scatterplots of the average experts' predictions with the observed static failure loads (table I). The data points were combined into different scatterplots (according to the protection device typology, geometry and material, the rock type, or combinations thereof) as shown as a reference example in figure 3. This Figure also provides an indication of the quality and, possibly, of the potential consequences of the predictions, which change according to the area of the scatterplot where the data-points are located.

\section{RESULTS}

\section{Pitons}

Pitons are anchor devices that can be placed inside cracks using a hammer. Generally, they are built with a blade (to be insert in the cracks) with a lug on one side for a karabiner to

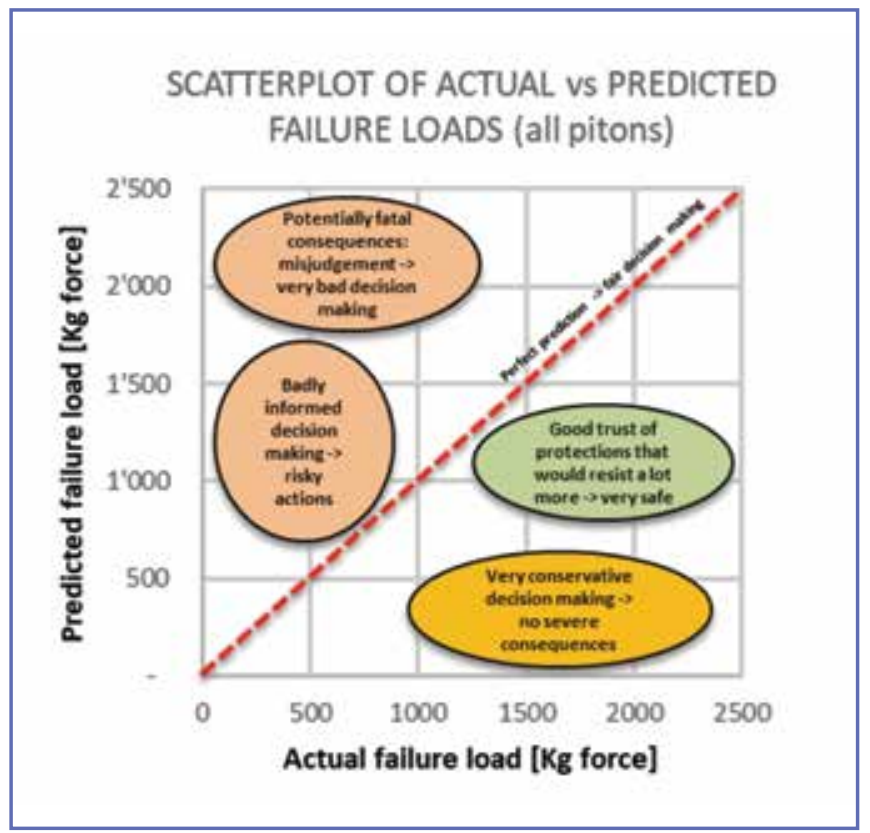

Figure 3. Example of a scatterplot used for categorizing the quality and the potential consequence of in field failure load predictions. 
be clipped in. EN 569 (9) requires tensile strength tests with a different loading direction, but with the pitons constrained in an "artificial" holding system. As far as the radial direction is concerned, the minimum values for the ultimate load are $25 \mathrm{kN}$ (for safety pitons, generally used for building stance) and $12.5 \mathrm{kN}$ (for progression pitons). Different configurations and types of pitons were tested. Pitons are usually built using soft steel and high carbon hardened steel. Generally, soft steel pitons should deform inside the crack, making them the common choice for soft rock, whereas hardened steel pitons are usually employed in regular cracks of hard rock such as granite. Both hard steel as well as soft metal alloy types have been tested in soft and hard rock. In order to verify the effect of the material used to manufacture pitons versus the type of rock, a variegate campaign of tests was carried out, thus, hard piton/hard rock - soft piton/soft rock - hard piton / soft rock - soft piton / hard rock tests were performed and the results herein exposed.

The most recurrent actual failure mode of pitons is a sort of slippage / pop out of the crack. Thanks to medium speed camera records, the collapse steps were observed: initially, a slight deformation of the piton occurs, followed by a medium-to-severe bearing destruction of the edges of the crack; finally, an abrupt piton pop out concludes the collapse, as shown in Figure 4. The measured ultimate load was scattered from about 6 up to $18 \mathrm{kN}$. Only in a few cases a mechanical collapse of the piton lug metal part was obtained. This was observed by a failure analysis showing a large permanent deformation of the main blade, and in a few cases, a failure in the section between the blade and the lug, see figure 4 .

As expected, soft metal alloy pitons exhibit higher plastic deformation whencompared to hard steel. However, soft pitons do not exhibit a lower collapse load, with respect to hard steel ones. The kernel density Figure 5 a) shows that soft pitons seem to offer higher resistance than hard pitons (regardless of their length and the rock type). Mountaineers commonly place hard pitons on hard rock. However, tests show very little difference in the ultimate load; besides, soft metal pitons show an increased capability to deform and to fit the internal shape of the crack also during load application. In the authors' opinion, thanks to the plastic deformation, this type of piton can withstand higher failure loads compared to the hard steel pitons. On the contrary hard steel pitons exhibit a much-reduced plastic deformation making them very suitable for re-use (that could be a key feature in the practical use).

Focusing on other aspects, long pitons exhibit a higher strength than short pitons (provided they are fully hammered into the rock), see figure $5 \mathbf{b}$ ). A deeper investigation of the differences (in strength) of pitons manufactured with different materials on Granite (hard rock) or Sandstone/Dolo- mite (soft rock) was carried out. For this purpose, several combinations of piton materials and rock types were tested. A summary of the results is reported in figure $5 \mathbf{c}$ ) (the results were collected for each type of rock) and are shown in more detail in figure $\mathbf{5} \mathbf{d}$ ). Ductile metal (soft) pitons placed in hard rock exhibit higher strength, even though this type of combination reduces the possibility to re-use the piton and most likely for this reason, soft pitons are commonly used just in soft rock. Hard metal pitons in hard rocks are likely to perform poorly due to their low capability to adapt. This low ability to deform results in the generation of only a few contact points with the hosting crack and consequently, an abrupt popping out can be expected once loaded. On the contrary, hard metal pitons perform well on soft rock.

With reference to the evaluation of the quality of the experts' predictions of the static failure loads, figure 6 summarizes the outcome of the analysis. More specifically, the scatterplot of all the piton failure loads predicted versus the actual failure loads of figure 6 (a) shows that, on average, the predictions are quite satisfactory- located in a quite concentrated region around the line indicating the perfect predictions. Interestingly, there is no bias towards either the conservative or the

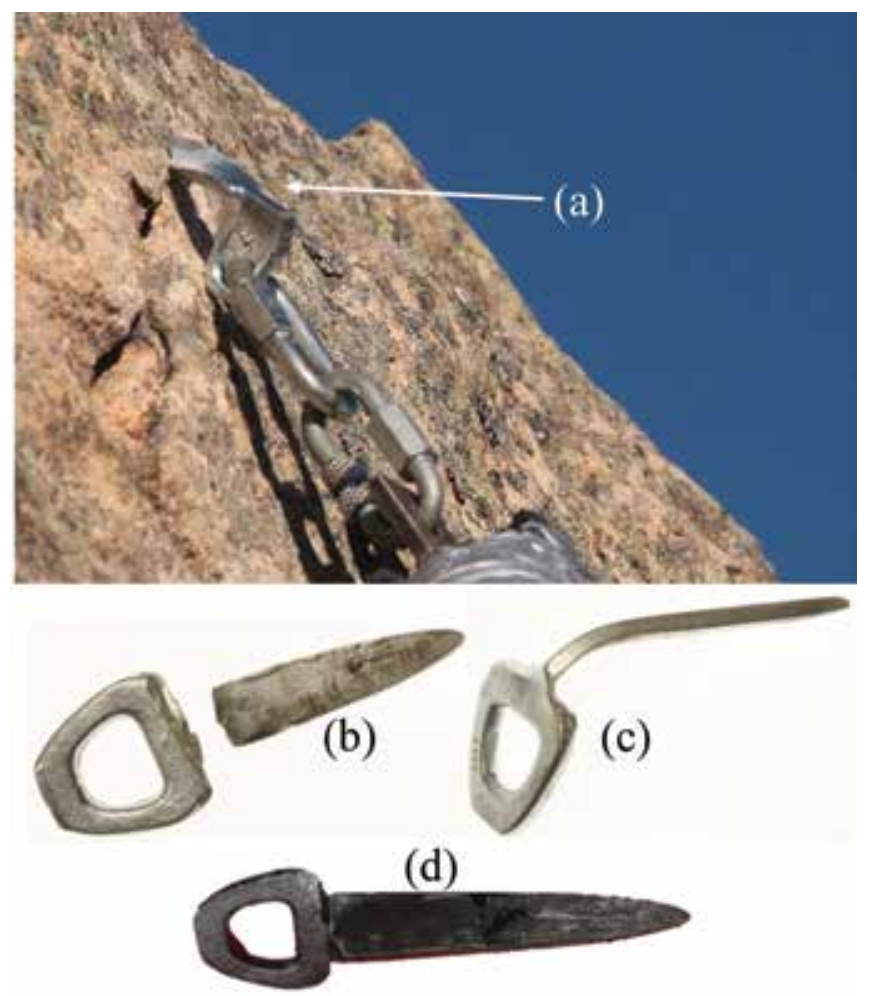

Figure 4. Failure mode of the pitons a) deformed soft piton placed in a crack pops out, b) ruptured soft piton, c) bent soft piton shaft, d) slightly deformed hard piton. 

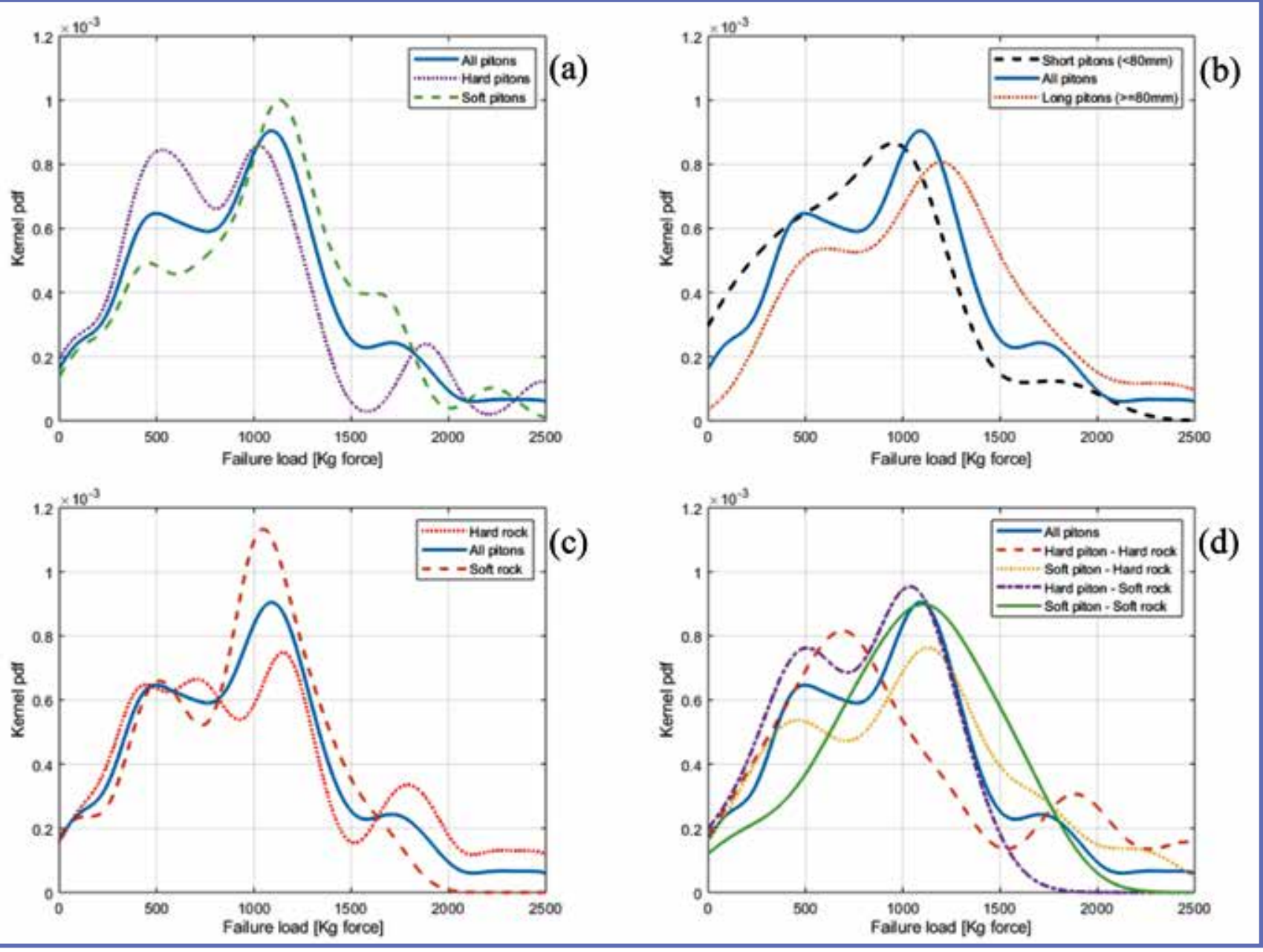

Figure 5. Kernel density of pitons (total of 69 observations available), an explanation of each figure is reported in the text.

non-conservative regions of the plot, but this is probably due to the nature of the experiment, which was testing the predictor's ability without any possible consequences on the predictor him/herself (in that case, much more conservative predictions are to be expected). However, a few outliers can be identified, both in the regions of conservative predictions, and in the region of dangerous predictions, although not in the very non-conservative area. In order to identify the motivations/ causes of these misjudgments, additional scatterplots were created, where only the predictions associated to either a specific piton material (hard and soft, figures $\mathbf{6}$ (b) and (c), respectively) or rock material (hard and soft, figures 6 (b) and (c), respectively) are reported. The analysis of these scatterplots shows that the outliers are only present in the predictions involving hard rocks, and not in those involving soft rock. At the same time, the piton material seems not to affect the presence of outliers in the predic- tions, thus confirming that the experts have more difficulties in predicting the resistance of pitons placed in hard rock or that the mechanical behavior of pitons in hard rock is more uncertain.

Chocks are special shaped nuts attached to a metal wire for placement and the load application by clipping a karabiner. EN 12270 (10) requires that chocks, tested in an "artificial holding" have to prove a failure load over $2 \mathrm{KN}$. Chocks are simply placed in the crack by hands, so are defined as "fast placement" anchors. All the placed chocks failed without leaving their hoisting crack. Generally, chocks fail at the metal wire, sometimes in the loop interfacing the karabiner body, or alternatively along the wire.

The failure mechanism observed, shown in figure 7, consists of practically no relative movement between the chock and the hosting crack, but involves rope loop elongation, initially breaking a single wire, followed by chain-collapse of the 


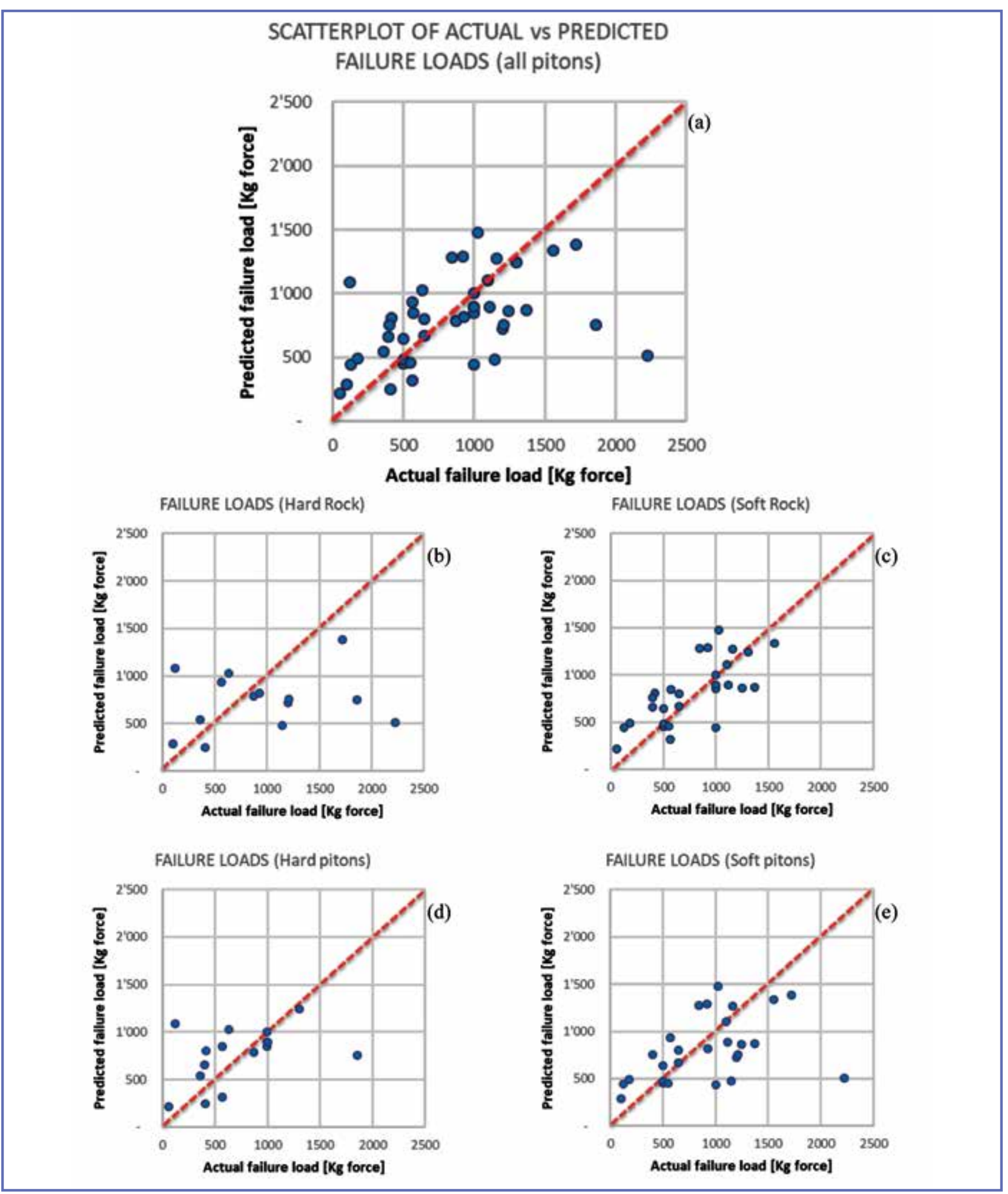

Figure 6. Evaluation of the experts' predictions, an explanation of each figure is reported in the text. Chocks, nuts. 

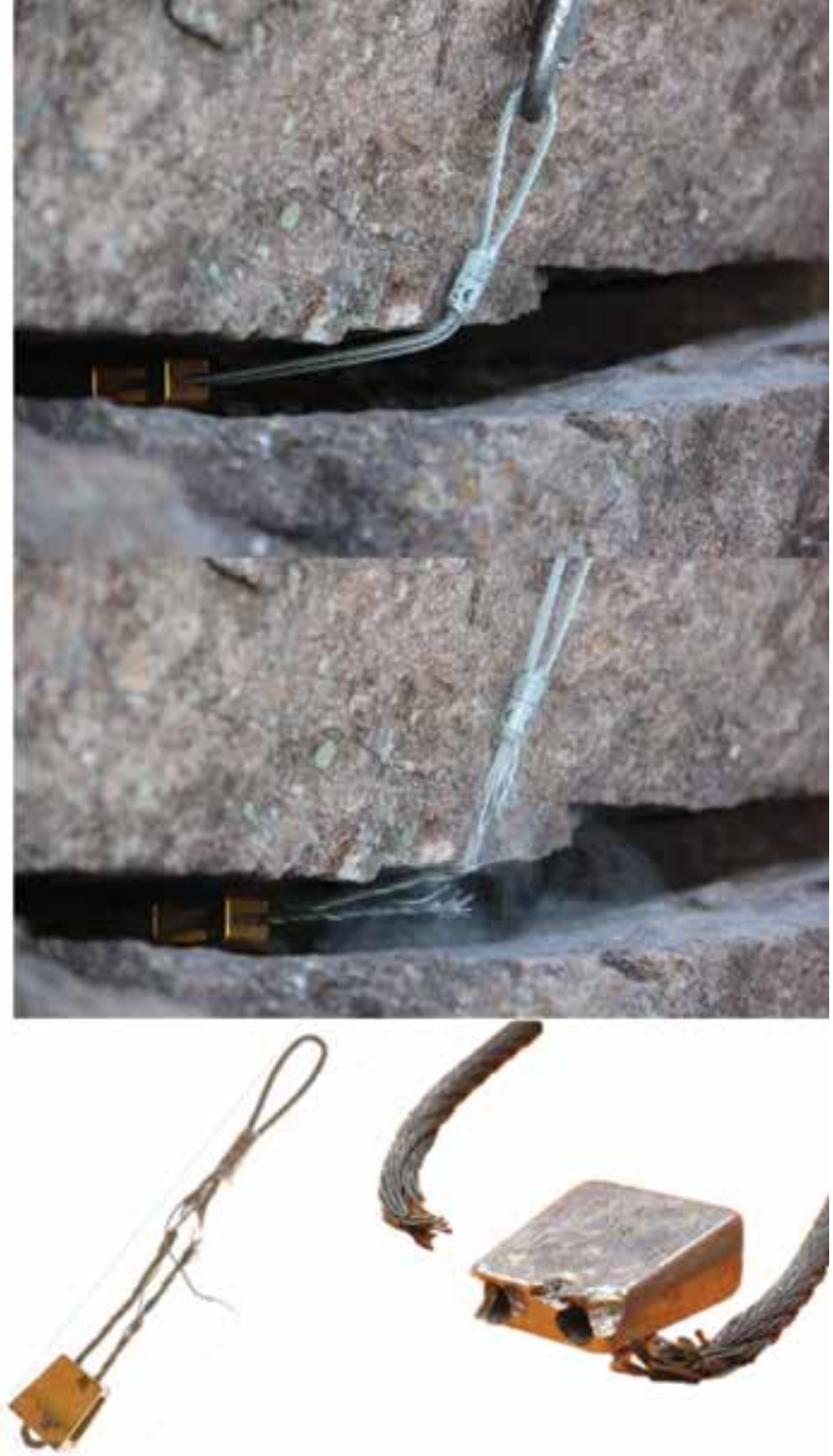

Figure 7. Failure mode of a chock / nut.

companion strand wire, up to a full separation, associated to a certain amount with unwinding of a single wire. The "post-mortem" analysis proved that chock-to-rock contact points were limited and localized to relatively small surfaces, as witnessed by the chock overall coloured chemical conversion, which remained in pristine condition. The measured ultimate load was scattered between about 6 up to $12 \mathrm{kN}$, as visible in figure 8 , thus, higher with respect to the requirement of the standard EN 12270 (10). It is worth mentioning that the spread of the strength is very high, ranging from a

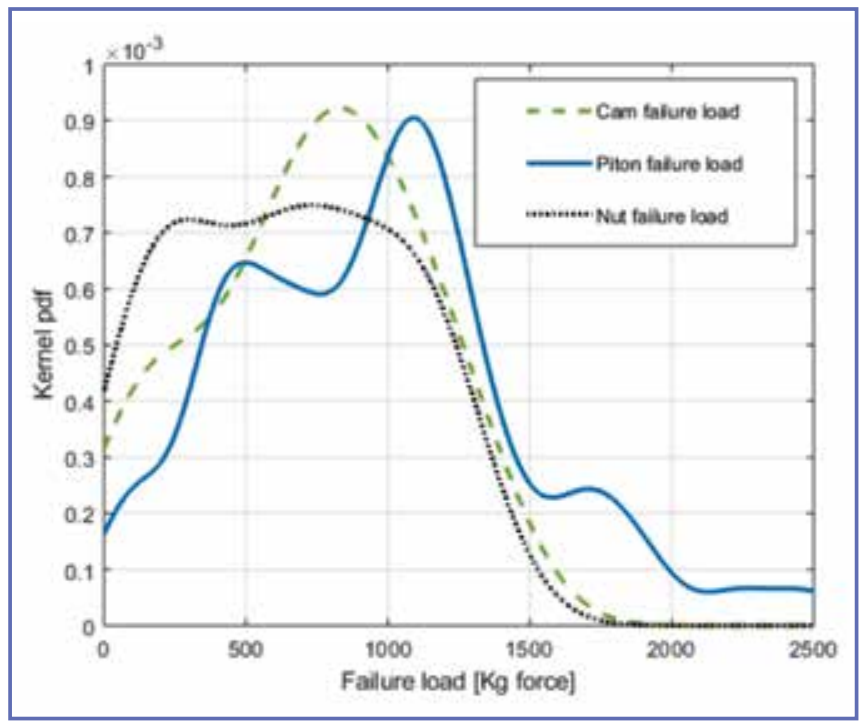

Figure 8. Kernel density of nuts (16 observations available) and cams (21 observations available), compared with pitons (69 observations available).

high value (close to the pitons strength) to a not negligible number of samples that fail at a very low level of loading. The strength of such a device is, in fact, very dependent on the placement.

The analysis of the experts' predictions by the scatterplot of figure 9 shows quite good agreement of the predictions with the actual static failure loads. Provided that the number of available observations and corresponding predictions is limited, a slight tendency to conservativeness can be noted for higher failure loads (i.e., probably, better placements), confirming a rather common misbelief of the climbing community that these kinds of passive protection device, when placed in the field, do not appear to be as reliable as they actually are. Indeed, the placement of these protection devices requires great care and much more experience than those required by other safety systems. Moreover, experts are somehow conscious of the level of strength of the device during placement, even if this strength is very reduced (figure 9 shows just one outlier with respect to this trend). This means that, in a real scenario, users may adopt some action in order to mitigate this possible lower strength, i.e. simultaneously placing two or more protections.

\section{Cam devices}

Cam devices belong, like chocks, to the group of "fast placement" devices. They are similar to chocks, but due to the incorporated spring system, each device can fit different crack sizes. EN 12276 (11) requires that cam devices (fric- 


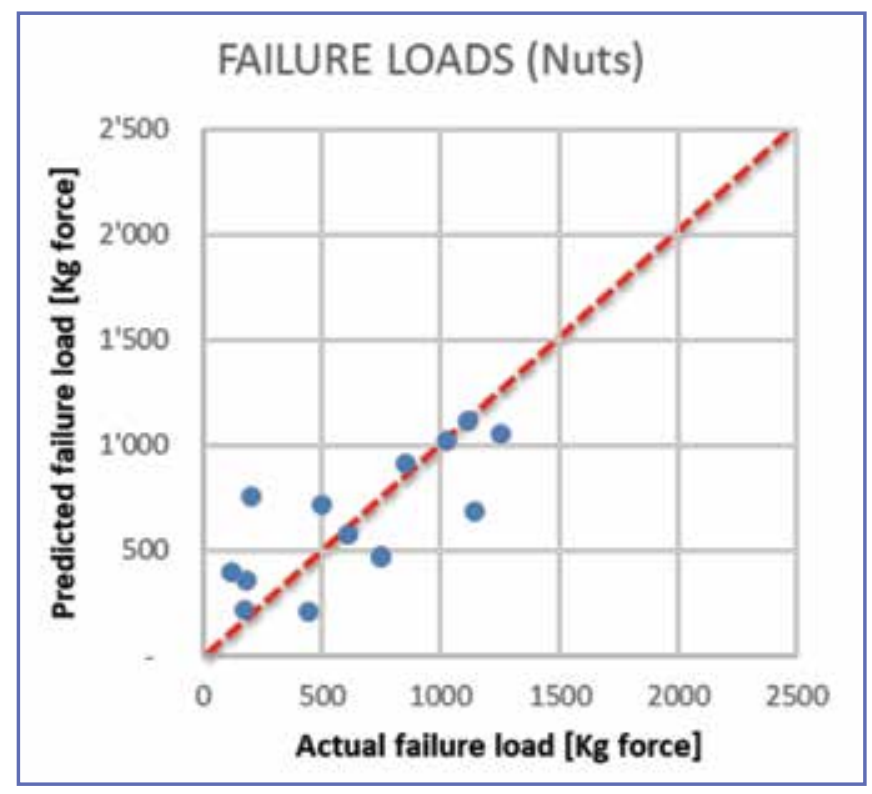

Figure 9. Evaluation of the experts' predictions for the nuts. tional anchor), tested in an "artificial holding", have to prove a failure load over $5 \mathrm{KN}$ (in two different positions). Due to the fact that their gripping action is obtained by means of friction, different shapes have been designed and built. The devices tested range from: a) old-fashioned solid bar models to b) modern wired body with built-in slings devices, while also testing c) early wired body models. Each of them shows a peculiar failure mode, as shown in figure 10. Model a) was characterized by snaking out of the hosting crack exhibiting ultra large permanent bending of the rigid bar or double shearing of the main shaft at both sides of the bar. Model b) popped out from the crack after a snaking settlement as the load increased. Model c) failed in the crimp as the wire loop slide out from it.

From the "post-mortem" analysis performed on the failed devices, it was observed that on model b) the only remarkable outcome are the limited scratches on the cam teeth. On the contrary, the other models showed obvious clues of the described failure mode. The measured ultimate load was scattered between 7 up to $14 \mathrm{kN}$, see Figure 8. Similar to the

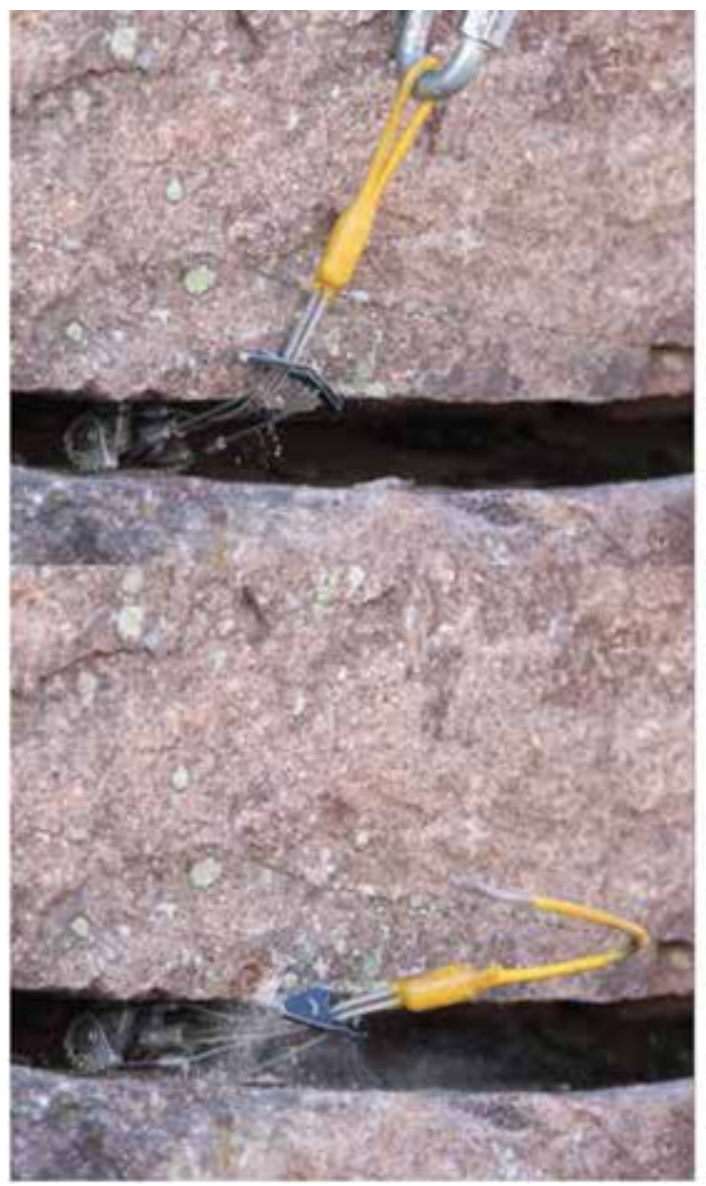

Figure 10. Failure mode of the cams.

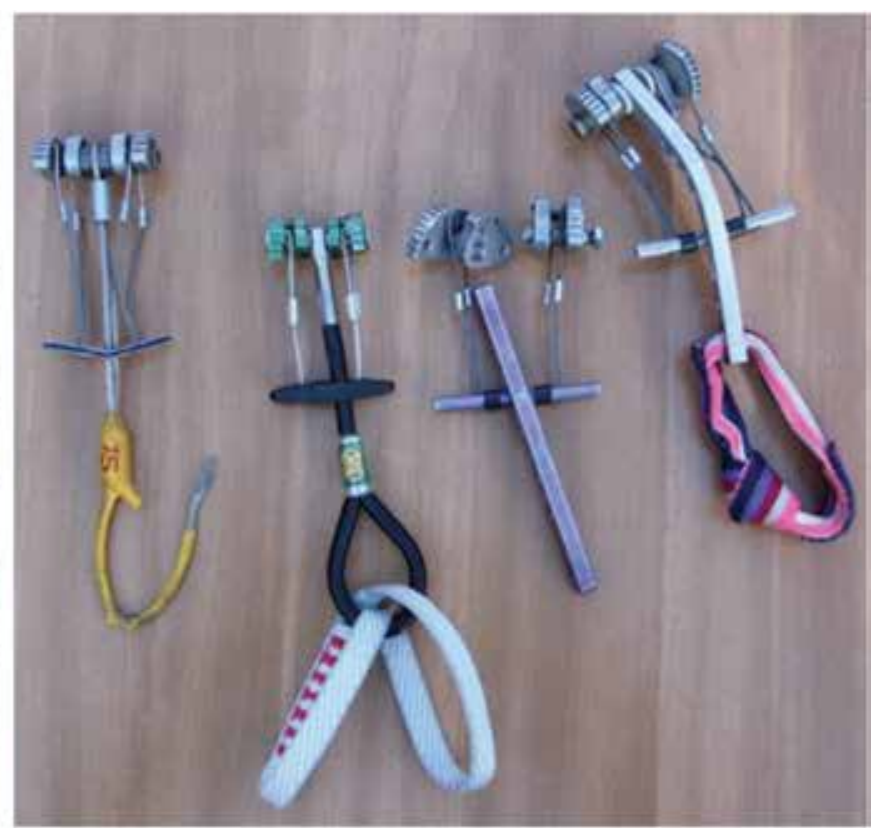


nuts, the failure load was higher with respect to the requirement of the standard EN 12276 (11).

As for the nuts, the spread of the strength was very high ranging from a higher value (close to the pitons strength) to a not negligible number of samples that failed a very low level of loading; however, very few specimens exhibited a very low strength. Such devices are, in fact, very dependent on the placement, too. Nonetheless, the possibility to adjust the placement by means of a spring that fits different crack sizes make the placement of such a device less critical than the nut placement.

The analysis of the experts' predictions by the scatterplot of figure 11 shows quite good agreement between the predictions and the actual static failure loads. Contrary to the previous case of the nuts, a tendency to be slightly non-conservative can be observed at low failure loads, which might confirm another common belief to over-trust the performances of cams, even when the placement is not very good (as is the case of low failure loads). Again, it should be noted that the number of observations and corresponding failure load predictions available for the analysis are limited, and further tests should be performed to confirm these behaviours.

\section{ASSESSMENT OF THE STRENGTH VERSUS LOAD}

With the aim to verify not only the strength of the protections, but also their capability to withstand the load applied

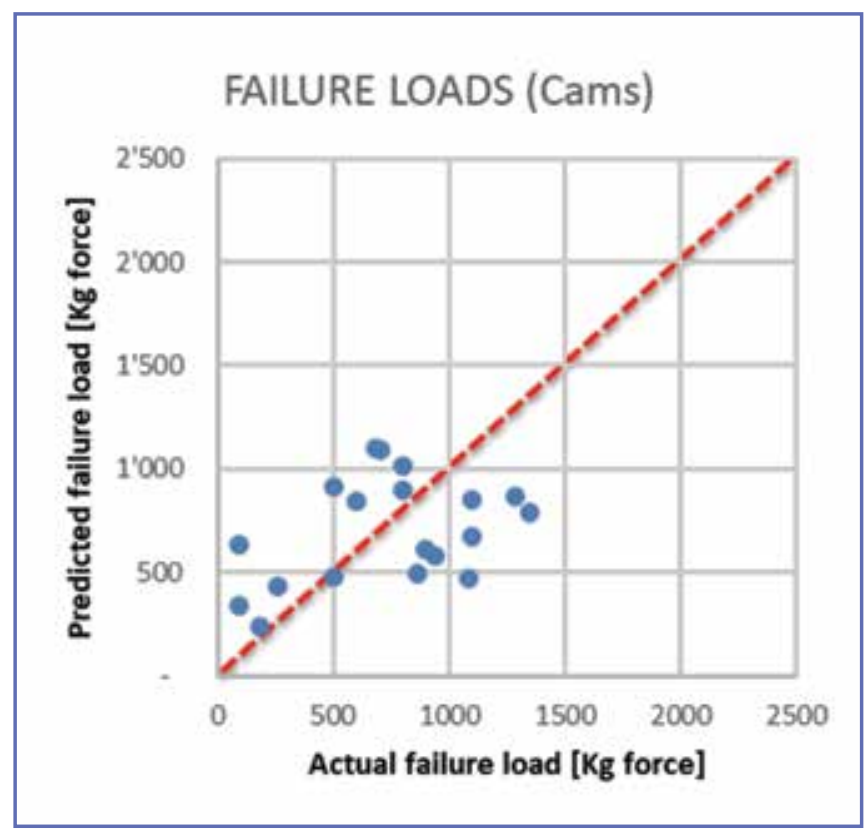

Figure 11. Evaluation of the experts' predictions for the cams. within the safety chain, a further investigation was carried out. As described above, dynamic tests were performed in a laboratory environment and in the field, but in a controlled setup. These tests were carried out separately with respect to the tests on the protections because they are aimed at measuring the actual load on the protection, but not its strength (in most of these tests, the protections used are dummy, characterized by very high strength). These tests involved dropping a weight, mimicking a fall of the climber from a given height and measuring the load on the last protection of the chain and/or on the belay stance. Also, in this case, the observed data was statistically processed by resorting to the kernel density approach. The comparison between the measured loads on the last protection of the chain and belay stance and the ultimate strength of the protections are shown in figure $\mathbf{1 2}$.

Indeed, safety would recommend strength higher than load, but this is not always possible due to the large spread of the strength, combined with that of the loads. In other words, the uncertainty affecting strength and loads are such that a finite probability exists that the protections fail when dynamically loaded by the fall of a climber. By properly manipulating the data available, it is possible to provide an estimate of this failure probability, i.e. the probability $P[$ Strengths-Loads $<0]$. It is important to state that even if the number of tests is considerable, they are not

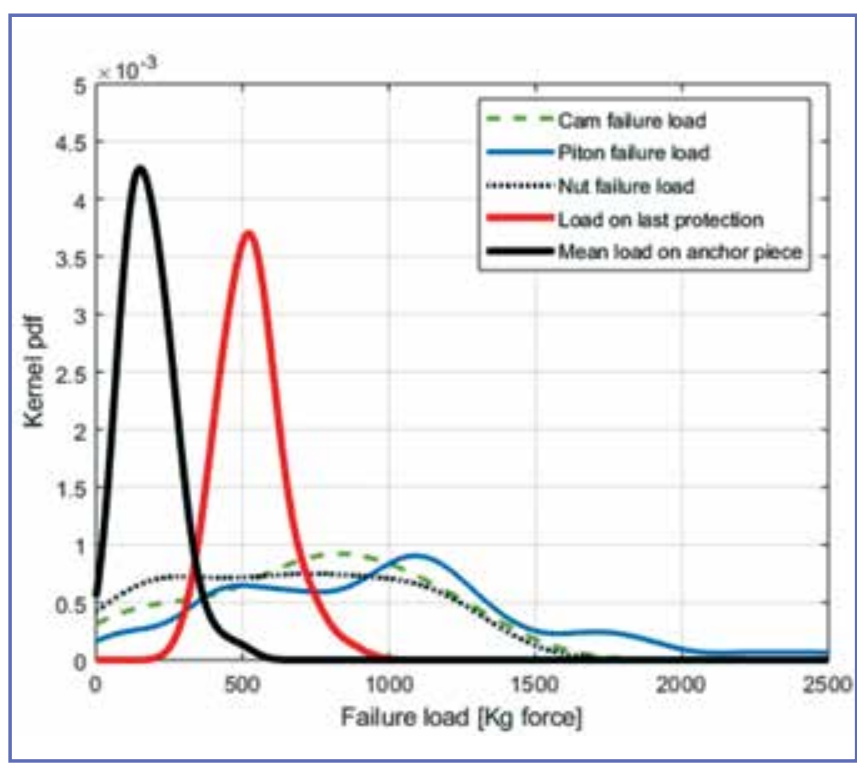

Figure 12. Comparison between kernel densities of strength of the anchors with respect to the kernel densities of the loads applied on the last protection (red, 310 observations available) and on the belay stance (black, 224 observations available). 
representative of all the possible cases that occur during actual climbing; in addition, a large spread is present in the data. Thus, the size of the observation population is not always optimal to draw robust conclusions and the statement of the present research has to be considered as an advice for the practice. Table II summarizes the results obtained for the different types of protections, considering the possible failure of the last protection of the chain and the failure of the belay stance. As expected, due to the dynamics of the load redistribution among all the elements of the climbing chain, the loads on the belay stance are much smaller than those acting on the last protection, so that the associated failure probability becomes significantly lower. Moreover, it is important to state that it is good practice to build a stance with two or more anchors. Even if the distribution of the load is not uniform on the multiple anchors (4), the load on a single anchor is lower. Therefore, results in figure $\mathbf{1 2}$ and table II can be considered as an upper limit for the stance in case the load is reacted by just one anchors-the worst case.

Despite this, the probability of failure of the stance is remarkably low, especially when pitons are used. This is comforting, as a safe belay stance allows a robust constraint of both climbers to the rock or ice wall.

\section{DISCUSSION}

Drawing a conclusion was difficult due to the large amount of scattering and the reduced protection population tested. The number of tests (106) and of predictions (approx. 1000) are not low per se, but the large variability of the protection types and of its usage make the size of the observation population not always optimal to draw robust conclusions. However, we were able to formulate some remarks.

As far as the piton actual strength is concerned, the knowledge gained through the field experiments confirms that the characteristics requested by EN specifications and rules are functional for product qualification purposes, but of very little use when defining the load holding capa-

Table II. Estimated failure probabilities according to the type of protection and its use (last protection of the chain or belay stance).

\begin{tabular}{lcc}
\hline & Last protection & Belay stance (worst case) \\
\hline Pitons & $28 \%$ & $6 \%$ \\
\hline Friends & $34 \%$ & $14 \%$ \\
\hline Nuts & $51 \%$ & $17 \%$ \\
\hline
\end{tabular}

bilities once the devices are in place. Spread is remarkable, however, even if the actual strength does not match the requirement of the standard, the comparison with the actual load applied is fairly encouraging. The probability of failure is important when all the type of investigated anchors are involved as the last protection in a fall, but, as expected, it is drastically reduced when they are used for building belay stances. This is especially true for pitons. As far as the investigation on the effect of the material of the pitons versus the material of the rock is concerned, pitons manufactured with ductile steel show a better behaviour in terms of strength, even if they have limited possibility to be re-used, which is clearly a drawback in the mountaineering practice. On the contrary, hard metal in hard rockscan sometimes produce poor performances because of its low capability to adapt; hard metal can generate only a few contact points with the hosting crack and consequently, an abrupt popping out is to be expected once loaded.

With regards to nuts, it is worth underlining the importance of a correct placement: when placed correctly, the actual strength achieved by the device in the field complies and is higher than the classification of the EN standard. This is fairly true in all the arrangements where the "obstacle" function is fulfilled; conversely, lower performance may be expected when the "friction" function (between the wedge-shaped block and the hosting crack) plays a predominant role. The cracks used were very suitable for nut placements, as confirmed by the high value of the failure load predicted by the evaluators. Similar behaviour was observed for cams. In this case better results were achieved with newly designed equipment: this suggests that outdated models should be retired from daily use by their owners.

The lesson learned from the experiments is that modern equipment (cams and pitons) shows a "fit for purpose" behaviour, not too dissimilar with respect to pitons, assuming of course a sound placement. Finally, the device-rock coupling dictates the pairs actual strength, thus, a correct choice and a correct placement are fundamental for all the equipment.

\section{CONCLUSIONS}

To the author's best knowledge, the present work represents the first attempt to investigate the human capabilities to assess the reliability of a protection placement in-field. This kind of analysis is very important, since in rock climbing, higher safety levels can be achieved only by properly combining improved designs of the protection devices with increased in-field awareness of their performances. This in turn, can only derive from in-depth inves- 
tigations of their physical functioning and interaction with the field environment, and of the consequent psychological implications on the climber's decision-making process. Finally, even if a limited number of available predictions has been obtained, they were sufficient to highlight some common misbeliefs in the climbing community, potentially leading to safety pitfalls and sub-optimal decision making.

\section{CONFLICT OF INTERESTS}

The authors declare that they have no conflict of interests. (12).

\section{REFERENCES}

1. Vogwell J, Minguez JM. The safety of rock-climbing protection devices under falling loads, Engineering Failure Analysis 2007; 14: 1114-1123.

2. UIAA 101 - Dynamic mountaneering ropes - Version previous February 2018.

3. EN 892. Mountaineering equipment - Dynamic mountaineering ropes - Safety requirements and test methods -2012 .

4. Bedogni V, Bressan G, Melchiorri C, Zanantoni C. Stances in mountaineering and climbing activities: an analysis and a proposal for an improved equalized anchoring, Sports Eng 2015; 18: 302-215. Doi: 10.1007/s12283-015-0177-3.

5. Hawksaw S. Strength and reliability of chemically bonded rock-climbing anchors in sandstone. The University of Sydney: Department of Civil Engineering 2003.

6. Karalis DG et al. "Failure analysis of a rock anchor made of stainless steel in marine environment" Engineering Failure Analysis 2012; 19: 123-130.

\section{ACKNOWLEDGMENTS}

We would like to acknowledge all the volunteers that participated actively in this research and specifically, all the members of the Safety Commission of the Italian Alpine Club (CAI). A special thanks to Massimiliano Avalle, Vittorio Bedogni, Gianfranco Biava, Giuliano Bressan, Gilberto Garbi, Elio Guastalli, Franco Lambri, Gianluigi Landreani, Giuseppe Milesi, Andrea Monteleone, Davide Rogora, Enrico Volpe, for their contributions in the activity and the Corps of the "Guardia di Finanza" for their hospitality at Passo Rolle, several schools of Mountaineering inside the CAI and several other volunteers that provided useful contributions in the execution of the tests.

7. Bedogni V, Manes A. An experimental investigation of the effect of the placement angle on the collapse of ice screw anchors, Engineering Failure Analysis, 2012; 26: 139-150.

8. Wand MP, Jones MC. Kernel Smoothing. London: Chapman \& Hall/CRC 1995.

9. EN-569 Mountaineering equipment. Pitons. Safety requirements and test methods 2007.

10. EN-12270 Mountaineering equipment. Chocks. Safety requirements and test methods 1988.

11. EN-12276 Mountaineering equipment. Frictional anchors. Safety requirements and test methods 1999 .

12. Padulo J, Oliva F, Frizziero A, Maffulli N. Muscles, Ligaments and Tendons Journal - Basic principles and recommendations in clinical and field Science Research: 2018 update. MLTJ 2018; 8(3): 305 - 307. 